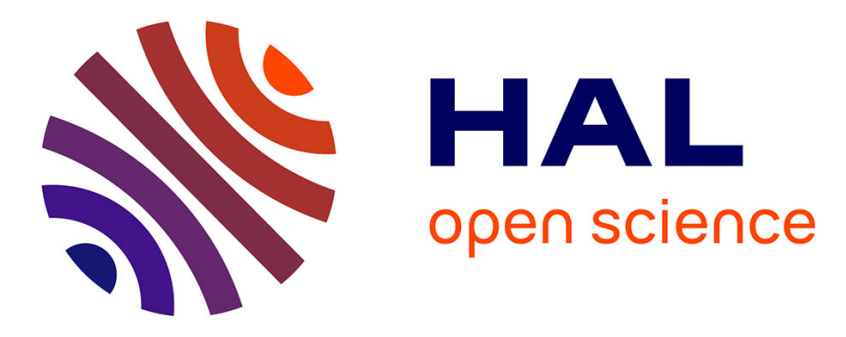

\title{
Spino-pelvic alignment influences disc hydration properties after AIS surgery: a prospective MRI-based study
}

Kariman Abelin-Genevois, Erik Estivalèzes, Jérôme Briot, Annick Sévely, Jérôme Sales de Gauzy, Pascal Swider

\section{To cite this version:}

Kariman Abelin-Genevois, Erik Estivalèzes, Jérôme Briot, Annick Sévely, Jérôme Sales de Gauzy, et al.. Spino-pelvic alignment influences disc hydration properties after AIS surgery: a prospective MRI-based study. European Spine Journal, 2015, 24 (6), pp.1183-1190. 10.1007/s00586-015-3875-4 . hal-01315354

\section{HAL Id: hal-01315354 \\ https://hal.science/hal-01315354}

Submitted on 13 May 2016

HAL is a multi-disciplinary open access archive for the deposit and dissemination of scientific research documents, whether they are published or not. The documents may come from teaching and research institutions in France or abroad, or from public or private research centers.
L'archive ouverte pluridisciplinaire HAL, est destinée au dépôt et à la diffusion de documents scientifiques de niveau recherche, publiés ou non, émanant des établissements d'enseignement et de recherche français ou étrangers, des laboratoires publics ou privés. 


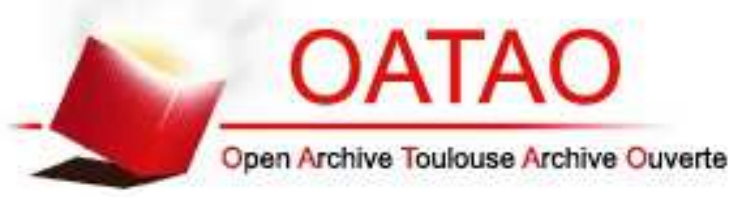

\section{Open Archive TOULOUSE Archive Ouverte (OATAO)}

OATAO is an open access repository that collects the work of Toulouse researchers and makes it freely available over the web where possible.

This is an author-deposited version published in : http://oatao.univ-toulouse.fr/ Eprints ID : 15760

To link to this article : DOI:10.1007/s00586-015-3875-4

URL : http://dx.doi.org/10.1007/s00586-015-3875-4

\section{To cite this version :}

Abelin-Genevois, Kariman and Estivalèzes, Erik and Briot, Jérôme and Sévely, Annick and Sales de Gauzy, Jérôme and Swider, Pascal Spino-pelvic alignment influences disc hydration properties after AIS surgery: a prospective MRI-based study. (2015) European Spine Journal, vol. 24 (n 6). pp. 1183-1190. ISSN 0940-6719

Any correspondence concerning this service should be sent to the repository administrator: staff-oatao@ listes-diff.inp-toulouse.fr 


\title{
Spino-pelvic alignment influences disc hydration properties after AIS surgery: a prospective MRI-based study
}

\author{
Kariman Abelin-Genevois ${ }^{1,2} \cdot$ Erik Estivalezes $^{2} \cdot$ Jerome Briot $^{2} \cdot$ Annick Sévely $^{3}$. \\ Jerome Sales de Gauzy ${ }^{2,4} \cdot$ Pascal Swider $^{2}$
}

\begin{abstract}
Purpose To analyze the disc hydration and volumetric changes of the intervertebral disc after scoliosis surgery depending on the sagittal spino-pelvic organization.

Methods We conducted a prospective MRI study in 45 patients with surgically treated adolescent idiopathic scoliosis (AIS) with a minimum 2-year follow-up. Fusion ended at L1 $(n=13), \mathrm{L} 2(n=14), \mathrm{L} 3(n=11)$ or L4 $(n=7)$. Total disc $(\mathrm{Vd})$ and nucleus volumes $(\mathrm{Vn})$ were extrapolated from $3 \mathrm{D}$ reconstruction using a custom-made image processing software (Biomechlab ${ }^{\circledR}$, Toulouse, France). Nucleus and external disc contours were semi-automatically detected on turbo spin echo T2-weighted sequence joined 3-mm sagittal cuts. Disc hydration was extrapolated from the nucleus-disc volume ratio ( $\mathrm{Vn} / \mathrm{Vd}$ ). Radiographic sagittal parameters were measured pre- and post-operatively on full spine standing views (pelvic incidence, sacral slope, L1S1 lumbar lordosis). Lumbo-pelvic congruity was calculated by the ratio LL/SS according to Stagnara.

Results Mean PI of the cohort was $55^{\circ}\left(34^{\circ}-85^{\circ}\right)$. After surgical correction, lumbar lordosis was slightly increased by $3^{\circ}(p=0.02)$ decreasing lumbo-pelvic congruity from
\end{abstract}

Kariman Abelin-Genevois

k.abelin-genevois@cmcr-massues.com

1 Department of Spine Surgery, CMCR des Massues, Croix Rouge Française, 92 rue Edmond Locard, 69322 Lyon Cedex 05, France

2 IMFT UMR CNRS 5502, Toulouse University, CHU Purpan, Toulouse, France

3 Division of Neuroradiology, Department of Radiology, CHU Purpan, Toulouse, France

4 Department of Pediatric Orthopaedic Surgery, Hôpital des Enfants, CHU Purpan, Toulouse, France
1.37 to $1.27(p<0.01)$. When pelvic incidence was less than $55^{\circ}$ (mean PI $46^{\circ}$ ), nucleus volumes have increased on average by $30 \%$ compared to the preoperative status in the unfused lumbar discs, while the total disc volumes has remained stable. Five-year follow-up $(n=13)$ confirmed the constant improvement of the disc hydration ratio. When PI was high (mean PI $64^{\circ}$ ), volumetric changes were very mild and significant changes in nucleus volumes and disc hydration ratio concerned only the intermediate lumbar levels (L2L3, L3L4 and L4L5).

Conclusions This prospective MRI study showed a significant and sustainable improvement in T2 hypersignal of the disc, indirectly indicating improvement of disc hydration content after AIS surgery. Analysis of disc volumetric changes according to the pelvic incidence suggests that these changes are under the influence of the sagittal spino-pelvic alignment. PI seems to play a key role in the homeostasis of the discs under fusion and should be taken into account for preoperative planning. The restoration of the lumbo-pelvic congruence may help to limit early degenerative changes in the free-motion segment discs after AIS surgery. Hydration content was less sensitive to surgery when PI was high, suggesting higher shear stress in the lower discs. Longer follow-up is required to confirm this hypothesis.

Keywords Adolescent idiopathic scoliosis $\cdot$ MRI $\cdot$ Spinal fusion - Intervertebral disc $\cdot$ Pelvic incidence $\cdot$ Disc hydration

\section{Introduction}

Spinal sagittal curvatures are specifically developed in humans together with sacrum verticalization as an answer to bipedalism. Ideally, the spino-pelvic organization is 
characterized by the correlation of the pelvic incidence (PI), an individually constant morphological parameter, and the sacral slope (SS) and pelvic tilt (PT), which are mathematically linked [1]. Moreover, lumbar lordosis (LL) is strongly correlated to sacral slope [2]. Stagnara emphasized the importance of the lumbo-pelvic congruity, i.e., the relation LL/SS [3]. Sagittal angles create the most suitable alignment to the pelvic morphology, to provide a stable and economic erect posture in terms of energy expenditure. In AIS, spinal sagittal alignment is abnormal. Somerville demonstrated the relative overgrowth of the anterior column, thus describing the scoliotic deformity as a rotational lordosis [4]. Main sagittal abnormalities are thoracic hypokyphosis and a moderate increase of LL. Thoracolumbar curves may induce thoracolumbar kyphosis. Spino-pelvic parameters remain strongly interrelated and comparable to normal population, except that the pelvic incidence is higher in AIS [5, 6].

Interest has been until now focused on the optimization of the sagittal correction, but did not explore the importance of the sagittal spino-pelvic organization in surgical planning and quality of correction. The surgical correction of the deformity, beyond stabilizing the deformity progression, aims the theoretical objective of halting the accelerated aging of the lumbar spine. The results of longterm historical cohort after spinal fusion with Harrington instrumentation have shown the importance to restore an adequate lumbar lordosis to prevent early degenerative changes and flat-back syndrome [7]. Modern instrumentation introduced in the 1980s by Cotrel and Dubousset brought to the forefront the sagittal contouring of the spine.

Intervertebral disc (IVD) is an avascular structure formed by the annulus fibrosus and an inner hydrated complex, the nucleus pulposus, characterized by high water content. IVD hydration, depending on proteoglycans content, is a leading determinant of the disc mechanical response to loading stress. In physiological conditions, nucleus pulposus exhibits viscous, fluid-like behavior, with an effective aggregate modulus. Loss of water content is one of the first events leading to disc degeneration [8]. Disc degeneration results in shear stiffness increase and decrease in aggregate modulus and loss of swelling pressure. Decreased hydration of NP results in decreased internal pressurization and disc height. These structural changes will result in transferring higher compressive loads to AF, compromising the disc function.

MRI is an accurate noninvasive tool that correlates signal intensity to water content in the nucleus [9]. Previous MRI studies on scoliotic patients have found mildto-moderate degenerative changes at the lumbar disc level, most using the Pfirmann score to define the degenerative changes. Green et al. [10] showed that twothirds of non-instrumented discs demonstrated mild signs of early disc degeneration one decade after AIS surgery. In order to improve our understanding of the postoperative behavior of IVD after spinal fusion, we conducted an MRI study based on 3D reconstruction of the disc to quantify the volume and hydration of the intervertebral disc [11]. According to the level of instrumentation, the nucleus/disc ratio, indirectly indicating hydration content of the disc, increased as a consequence of nucleus size increase and hydration uptake, suggesting that the correction of scoliosis modified the lumbar disc homeostasis. However, to understand what factors influence the future of the free segment below spinal fusion, we have continued the prospective follow-up and monitored the radiological parameters of the AIS cohort. We designed the present study to investigate the influence of the spinopelvic morphology on the behavior of lumbar IVD in a cohort of surgically treated AIS. The main hypothesis is that the pelvic morphology influences disc properties and biology after surgical correction of AIS.

\section{Methods}

The protocol of the study was initially approved by the Institutional Review Board of the Ethic Committee. We consecutively included patients with AIS candidate to surgery, who were scheduled in our institution between 2005 and 2007.

Forty-five patients (40 males, 5 females) achieved the complete follow-up (2 years minimum) with prospectively collected clinical, radiological and MRI data. Mean age at surgery was $15.1 \pm 1.7$ years. Magnetic resonance imaging (MRI) was performed after informed consent before and during the postoperative follow-up (at least 2 years after surgery). Posterior instrumented fusion using multisegmental instrumentation was performed by one experienced spine surgeon in the same institution.

Long cassettes standing antero-posterior and lateral radiographs of full spine were performed preoperatively. Major curve magnitude was measured according to the Cobb method on AP radiographs. Curve flexibility was assessed in laying position, by right and left side-bending radiographs. Inferior limit of fusion was decided according to the first most symmetrical disc on the side bending radiographs. The most cephalad and symmetrically loading disc was chosen as the inferior limit of the construct.

The following radiographic spino-pelvic parameters were measured on lateral views by an independent experienced observer (spine surgeon, PhD student) (Fig. 1): lumbar lordosis (LL), sacral slope (SS) and pelvic incidence (PI). Pre- and post-operative values of the spinopelvic parameters were compared with two-tailed Student's $t$ tests. The relation between all spinal and pelvic 
parameters was assessed using Pearson coefficients. The level of significance was set at 0.05 .

\section{IVD volumetric analysis}

Each AIS patient enrolled in the prospective study underwent a standardized MRI protocol before surgery, 3 months after surgery and at least 2 years after surgery. To minimize the bias of diurnal variations of disc height and nucleus size, MRI examinations were scheduled in the morning (patients were called at the latest at 8:00 am) and patients were asked not to have any physical activity. Acquisitions of the full spine were performed using a turbo spin echo T2-weighted sequence in the sagittal plane on a Magneton Vision 1.5 T. (joined cuts of $3 \mathrm{~mm}$ ). Disc volumetry was extrapolated using custom-made image processing software developed with Matlab $^{\circledR}$ (Mathworks ${ }^{\circledR}$ ). The process is detailed in Fig. 2. Using our new software Biomechlab ${ }^{\circledR}$ (Laboratoire de Biomécanique, Toulouse, France), a semi-automatic detection of the contours of each lumbar disc was performed. The external limit of annulus fibrosus and nucleus

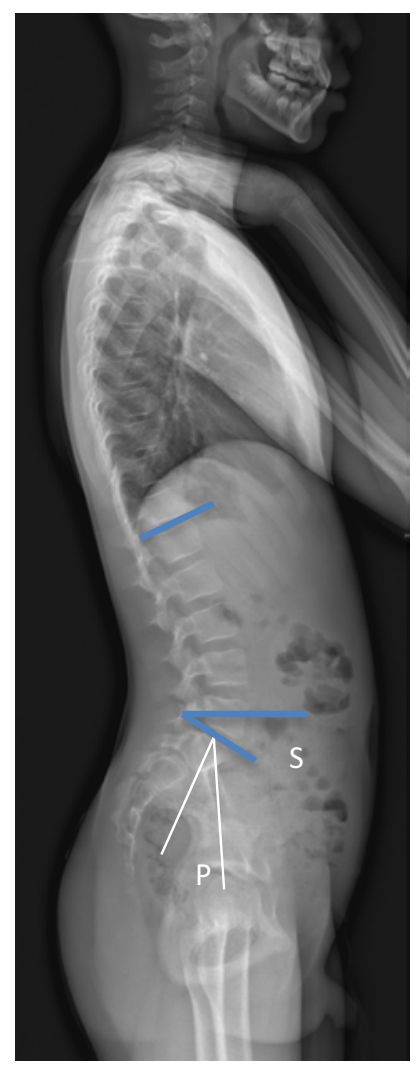

Fig. 1 Spino-pelvic parameters measurement on standing sagittal view of the spine. Lumbar lordosis (LL) is measured between the superior endplate of L1 and S1. Sacral slope (SS) is measured between the horizontal line and the sacral plateau. Pelvic incidence (PI) is measured between the perpendicular line to the sacral plateau and the line passing by the midline of the center of femoral heads pulposus were contoured on each sagittal slice by an independent spine surgeon. Nucleus pulposus was identified as the most hydrated inner complex (T2 high-intensity signal). The contrast was adjusted according to the cerebro-spinal fluid in order to detect the most hydrated part of the disc. Each lumbar disc was composed of approximately 10 sagittal cuts. After 3D volume reconstruction, volume properties of the total disc and the nucleus were extracted at each unfused lumbar level. Volumetric values were analyzed postoperatively in comparison to the preoperative one and served as reference as no normative values were available at the time of our study.

Total disc and nucleus volumetric changes $(\Delta \mathrm{Vd}, \Delta \mathrm{Vn})$ were analyzed according to the preoperative pelvic incidence. Patients with PI inferior to the average PI value of the cohort $\left(55^{\circ}\right)$ were considered as low PI group. Patients with PI equal or superior to $55^{\circ}$ were considered as high PI group. Inside high PI subgroup, a second analysis compared groups according to the extent of spinal fusion. Constructs ending at L1 or L2 level were defined as short fusion subgroup. Constructs ending down to L3 or L4 were considered as long fusion subgroup. Comparisons between pre- and post-operative disc and nucleus volumes were assessed by Student's $t$ test (level of significance, $p<0.05$ ).

\section{Results}

Mean preoperative curve magnitude was $48^{\circ}\left(36^{\circ}-89^{\circ}\right)$. Mean curve correction was $62 \%(36-81 \%)$.

Prior to surgery, mean LL was $-57.6^{\circ} \pm 11.2^{\circ}$. The pelvic parameters were measured at $55.2^{\circ} \pm 11.3^{\circ}$ for the $\mathrm{PI}, 42.8^{\circ} \pm 10.9^{\circ}$ for the SS. LL was moderately increased after spinal fusion $\left(+3^{\circ}, p=0.02\right)$. PI and SS remained unchanged. The correlation between LL and SS was weaker after surgery (LL/SS $=1.27 ; r=0.72, p<0.01$ ) than preoperatively $(\mathrm{LL} / \mathrm{SS}=1.37 ; r=0.59, p=0.02)$ (Table 1).

Spino-pelvic parameters were separately analyzed according to the length of instrumentation (Table 2). In both surgical groups, pelvic parameters remained unchanged. Correlation between LL and SS was higher in the short fusion group (preoperative correlation 0.78, $p<0.001$; postoperative correlation $0.62, p=0.007$ ) than the long fusion group (preoperative correlation 0.65, $p=0.004$; postoperative correlation $0.55, p=0.016$ ) and decreased postoperatively in both groups.

\section{IVD volumetric changes}

Disc volumetric changes were analyzed at each unfused lumbar level and results are presented separately according to the preexisting pelvic morphology (low or high pelvic 

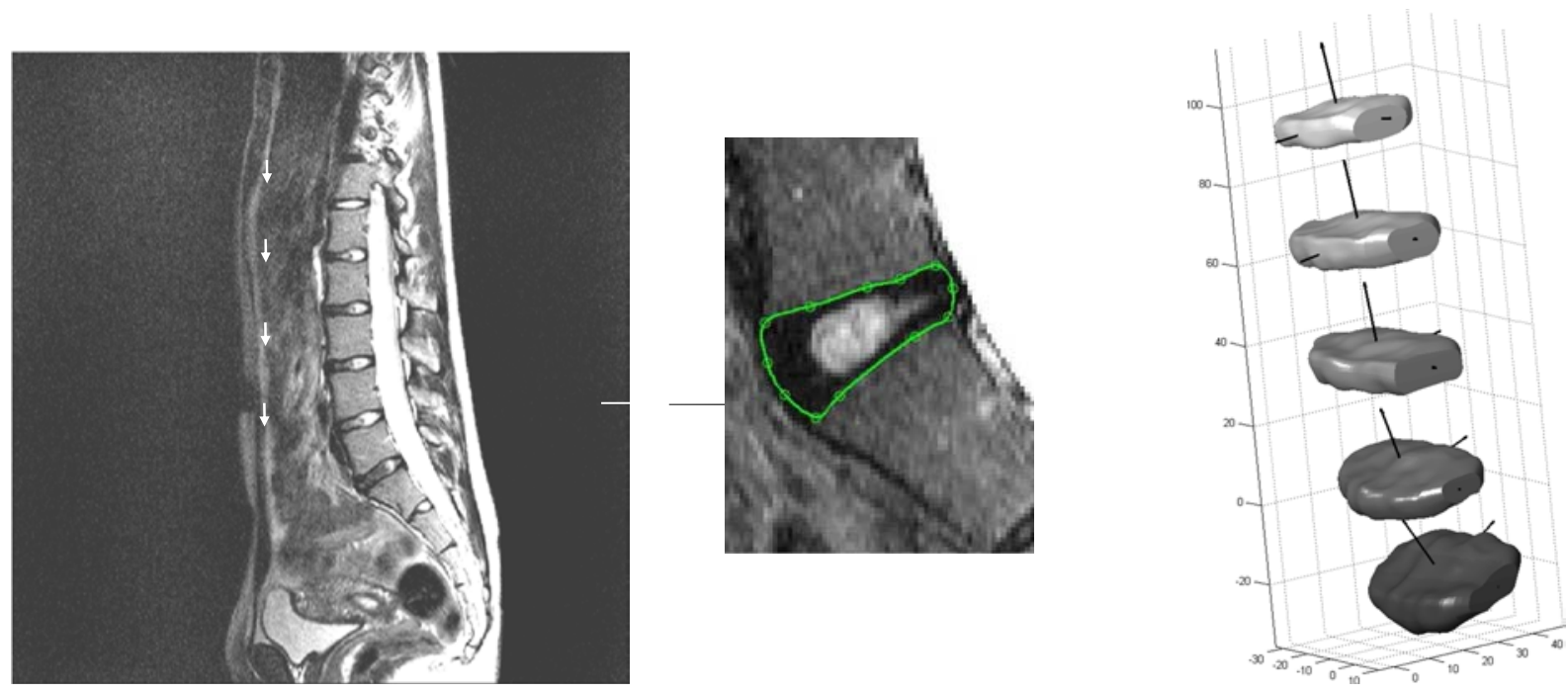

MRI Sagittal T2 weighted sequences

(DICOM images)

$\approx 10$ cuts/ disc level

Semi-automatic contouring of

annulus fibrosus (outer part of the disc)

nucleus pulposus (inner hydrated complex)

Fig. 2 3D reconstruction of the intervertebral disc from MRI T2 sagittal sequences

Table 1 Spino-pelvic parameters changes after AIS surgical correction

\begin{tabular}{llll}
\hline & $\begin{array}{l}\text { Preoperative } \\
\text { values }\end{array}$ & $\begin{array}{l}\text { Postoperative } \\
\text { values }\end{array}$ & $p$ value \\
\hline PI & $55.2^{\circ}+11.3^{\circ}$ & $55.3^{\circ}+11.0^{\circ}$ & NS \\
LL (L1S1) & $-57.6^{\circ} \pm 11.2^{\circ}$ & $-59.9^{\circ} \pm 11.8^{\circ}$ & 0.022 \\
SS & $43.2^{\circ} \pm 10.8^{\circ}$ & $44.4^{\circ} \pm 9.4^{\circ}$ & $\mathrm{NS}$ \\
LL/SS & $1.37 \pm 0.2$ & $1.27 \pm 0.2$ & $\mathbf{0 . 0 0 7}$ \\
Pearson & $r=0.72$ & $r=0.59$ & \\
correlation & & & \\
\hline
\end{tabular}

Bold value indicates statistically significant

incidence). Subgroups were comparable in terms of age, initial Cobb angle and frontal balance (main curve correction, lumbar disc wedging and CSVL deviation).

The low PI group included 24 patients; all of them were followed 2 years or more. Mean PI was $46.1^{\circ}\left(34^{\circ}-54^{\circ}\right)$. Fusion ended on L1 in eight patients, L2 in seven patients, L3 in nine patients and L4 in five patients. Table 3 summarizes disc $(\mathrm{Vd})$ and nucleus volumes $(\mathrm{Vn})$ variations quantified on 3 months, 2 years and 5 years postoperative MRI. Total disc volumes (Vd) were not modified by the surgical correction. Nucleus size significantly increased after 2 years in all free-motion lumbar levels except the
Table 2 Influence of lumbar fusion extent on spino-pelvic parameters

\begin{tabular}{lrlrl}
\hline & \multicolumn{2}{l}{ Short fusion } & \multicolumn{2}{l}{ Long fusion } \\
\hline PI & $55.8^{\circ}$ & & $55.2^{\circ}$ & \\
LL preop & $-57.7^{\circ}$ & & $-56.6^{\circ}$ & \\
Postop & $-53.9^{\circ}$ & $p=0.047^{*}$ & $-55.9^{\circ}$ & $p=0.43^{*}$ \\
SS preop & $42.1^{\circ}$ & & $43.6^{\circ}$ & \\
Postop & $43.7^{\circ}$ & $p=0.28^{*}$ & $44.0^{\circ}$ & $p=0.37^{*}$ \\
LL/SS preop & 1.42 & & 1.34 & \\
Postop & 1.26 & $p=0.006^{*}$ & 1.30 & $p=0.39^{*}$ \\
Pearson correlation & 0.78 & & 0.65 & \\
Coefficient & 0.62 & & 0.55 & \\
\hline
\end{tabular}

* Paired Student's $t$ test. Significance level set at 0.05

first lumbar disc (L1L2, $n=8$ ). Data at 5 years were available for 13 patients. They showed a marked increase of nucleus volumes at each level. Nucleus volumes increased at least by $+30 \%$.

The high PI group included 21 patients; all were followed at least 2 years postoperatively. Fusion ended on L1 in six patients, L2 in seven patients, L3 in seven patients and on L4 in one patient. Again, total disc volumes did not significantly change after the surgery. Two years after surgery, 
Table 3 Volumetric changes of the nucleus ( $\mathrm{Vn})$ and the disc $(\mathrm{Vd})$ in the unfused lumbar spine in the low PI subgroup (pelvic incidence $<55^{\circ}$ )

\begin{tabular}{ccccccc}
\hline & 3 months PO $(\%), n=24$ & $p$ value* & 2 years PO $(\%), n=24$ & $p$ value* & 5 years PO $(\%), n=13$ & $p$ value* \\
\hline DVd & & & & & & \\
L5S1 & 1.5 & 0.38 & 6.7 & 0.49 & -8.3 & 0.21 \\
L4L5 & -0.1 & 0.34 & 6.0 & 0.43 & -1.1 & 0.43 \\
L3L4 & 0.2 & 0.34 & 3.3 & 0.28 & 10.5 & 0.14 \\
L2L3 & -4.5 & 0.47 & -0.5 & 0.45 & -1.9 & 0.25 \\
L1L2 & 0.8 & 0.30 & 10.6 & 0.31 & 8.8 & \\
DVn & & & & & \\
L5S1 & 3.8 & 0.28 & 40.1 & $\mathbf{0 . 0 1 1}$ & 31.5 & $\mathbf{0 . 0 1 1}$ \\
L4L5 & 4.9 & 0.40 & 32.4 & $\mathbf{0 . 0 0 2}$ & 41.3 & $\mathbf{0 . 0 0 1}$ \\
L3L4 & 12.0 & 0.13 & 26.2 & $\mathbf{0 . 0 0 2}$ & 30.1 & $\mathbf{0 . 0 1 1}$ \\
L2L3 & -2.9 & 0.46 & 16.2 & $\mathbf{0 . 0 4}$ & 33.3 & $\mathbf{0 . 0 0 8}$ \\
L1L2 & -11.4 & 0.42 & 0.0 & 0.22 & 32.7 & $\mathbf{0 . 0 0 5}$ \\
\hline
\end{tabular}

* Preoperative values served as reference as no reference data exist in the normal population. $p$ values are given by Pearson test between pre- and post-operative values at each follow-up period ( 3 months, 2 years and 5 years)

Bold values indicate statistically significant

Table 4 Volumetric changes of the nucleus $(\mathrm{Vn})$ and the disc $(\mathrm{Vd})$ in the unfused lumbar spine in the high PI subgroup (pelvic incidence $\geq 55^{\circ}$ )

\begin{tabular}{ccccccc}
\hline & 3 months PO $(\%), n=21$ & $p$ value & 2 years PO $(\%), n=21$ & $p$ value & 5 years PO $(\%), n=5$ & $p$ value \\
\hline DVd & & & & & & 0.15 \\
L5S1 & -2.8 & 0.40 & 5.1 & 0.40 & -13.5 & 0.37 \\
L4L5 & -2.7 & 0.28 & -3.8 & 0.44 & -5.5 & 0.39 \\
L3L4 & -2.4 & 0.35 & 0.0 & 0.28 & -4.7 & 0.22 \\
L2L3 & 2.5 & 0.43 & 16.9 & 0.44 & -2.5 & NA \\
L1L2 & 10.9 & 0.48 & 11.8 & 0.36 & & 0.36 \\
DVn & & & & 0.06 & 7.8 & $\mathbf{0 . 0 1}$ \\
L5S1 & 1.2 & 0.44 & 14.3 & $\mathbf{0 . 0 1}$ & 21.0 & $\mathbf{0 . 0 2}$ \\
L4L5 & 6.8 & 0.15 & 17.9 & $\mathbf{0 . 0 2}$ & 32.9 & $\mathbf{0 . 0 4}$ \\
L3L4 & 4.3 & 0.32 & 17.6 & 0.08 & 30.9 & NA \\
L2L3 & 5.8 & 0.26 & 16.8 & 0.17 & & \\
L1L2 & 9.4 & 0.37 & 24.3 & & \\
\hline
\end{tabular}

Bold values indicate statistically significant

nucleus volumes increased at L2L3 and L3L4 but not in the distal parts of the lumbar lordosis (L1L2 L4L5 and L5S1) (Table 4). Nucleus volume varied differently according the extent of the fusion. Table 5 shows the volumetric changes when short fusion is performed on a high PI morphology. The nucleus volume increased in the lower levels (1 or 2 levels below the inferior instrumented vertebra). Comparatively, nucleus volume increase were significantly lower in the subgroup with long fusion and high PI (Table 6), where moderate significance was only reached in the intermediate disc level L4L5 $(+33.5 \%, p<0.05)$.

\section{Discussion}

Longitudinal MRI quantification of disc volumetric properties after surgical correction of AIS showed a marked increase in the nucleus volumes within 2 years while total disc volumes remained unchanged. These results suggest a process of disc remodeling, independent of geometric remodeling induced by the surgical correction of the spinal deformity. Nucleus volume increase, directly linked to T2 hypersignal, accurately reflects the disc hydration status. $\mathrm{Vn}$ increase between pre- and post-operative period strongly suggests improvement of IVD hydration. We assume that this is related to the modification of the loading stress exerted on the discs, especially by a repositioning of the nucleus within the disc. Surgical correction of AIS, by reducing the disc wedging, especially at the apex of the lumbar lordosis, apparently improved functional conditions of the disc under loading stress. According to Setton, mechanical factors influence the biological response of the IVD cells [12]. IVD cells are highly sensitive to differences in cells and tissue (collagen fibers) geometry. The primary stimuli in NP cells are fluid pressurization and volume change. Accadbled et al. [13] assumed that static 
Table 5 Volumetric variations after selective fusion (LIV at L1 or L2)

\begin{tabular}{lclll}
\hline & 3 months PO $(\%), n=13$ & $p$ value & 2 years PO $(\%), n=12$ & $p$ value \\
\hline DVd & & & & \\
L5S1 & 3.7 & 0.30 & 2.1 & 0.42 \\
L4L5 & 2.6 & 0.30 & 4.0 & 0.39 \\
L3L4 & 2.4 & 0.36 & 0.1 & 0.41 \\
L2L3 & 9.0 & 0.33 & 9.8 & 0.46 \\
L1L2 & 13.9 & 0.50 & 11.8 & 0.37 \\
DVn & & & & \\
L5S1 & 10.3 & 0.36 & 21.2 & $\mathbf{0 . 0 3}$ \\
L4L5 & 0.6 & 0.40 & 12.7 & $\mathbf{0 . 0 1}$ \\
L3L4 & 5.9 & 0.49 & 17.6 & 0.07 \\
L2L3 & 5.8 & 0.43 & 11.6 & 0.16 \\
L1L2 & 9.4 & 0.39 & 24.3 & 0.35 \\
\hline
\end{tabular}

Bold values indicate statistically significant

\begin{tabular}{ccccc}
\hline & 3 months PO $(\%), n=8$ & $p$ value & 2 years PO $(\%)$ & $p$ value \\
\hline DVd & & & & \\
L5S1 & -1.1 & 0.40 & 26.7 & 0.20 \\
L4L5 & -2.8 & 0.37 & -3.0 & 0.23 \\
L3L4 & -2.5 & 0.40 & 0.1 & 0.26 \\
DVn & & & & 0.25 \\
L5S1 & -15.6 & 0.40 & -6.4 & $\mathbf{0 . 0 4}$ \\
L4L5 & 20.6 & 0.03 & 33.5 & 0.42 \\
L3L4 & 0.9 & 0.36 & 17.3 & \\
\hline
\end{tabular}

Bold values indicate statistically significant
Table 6 Volumetric variations after long AIS fusion (LIV at L3 or L4) levels. In the low PI subgroup, AIS surgery improved both nucleus volume and hydration content at all lumbar levels. When PI is high, the ideal sagittal spinal shape is more curved [1], surgical correction of AIS may have created a relative loss of lordosis and increase the constraints exerted on the remaining lumbar discs. As a consequence, only moderate remodeling effect was observed. AIS patients with high PI require larger sagittal correction, which may be challenging when initial curve is severe and stiff.

Ideal biomechanical conditions are reached after selective fusion with low PI. Assuming that the scoliotic discs have a biomechanical dysfunction proportionate to the severity of the curvature and their position relative to the apex [14], the amount of correction required to induce significant and durable disc modifications is as higher as pelvic incidence is greater.

In AIS, spino-pelvic alignment differs from the normal population. Our results are similar to those from Roussouly et al. [6]: PI is higher, SS is higher and pelvis is more anteverted than normal population. LL is strongly correlated to SS. After surgical correction, parameters remained in the range of the preoperative values. by rebalancing the disc asymmetry, may induce better volumetric changes and disc rehydration at all lumbar 
Significant differences in the biomechanical behavior of the lumbar spine were observed depending on the pelvic incidence. Our results demonstrated that in low PI group, nucleus volume and disc hydration significantly improved in the long-term follow-up. Remodeling was effective for the lowest segments (lower arc of LL). In this subgroup, volumetric data 5 years or more after arthrodesis showed that remodeling was a constant and durable phenomenon, at all unfused lumbar discs. The highest improvement was observed in L5S1 level. Patients with high PI showed more modest volumetric and hydration changes compared to low PI group. These changes occurred only in the intermediate unfused lumbar segments (L2L3, L3L4). Volumetric changes of L5S1 were heterogeneous and not significant, probably reflecting stronger mechanical stress. Galbusera et al. [15] found sagittal parameters to influence spinal lumbar loads and muscle activity. Among them, LL was found to have a high relevance in determining the shear loads in comparison with the compressive loads. In this paper, the authors did not argument on the pelvic incidence. We can assume that for patients with a high PIinvolving a higher SS-theoretical LL should be more important to maintain the lumbo-pelvic congruity. Therefore, high PI should be related to higher shear stress after long fusion above the lumbar spine as it is in spondylolisthesis [16-18]. Whereas for short fusion, maximum mechanical stresses are absorbed by the adjacent segment allowing better hydration two levels under the fusion (including L5S1). Thus, in idiopathic scoliosis, it appears that the sagittal organization may have a leading role in the biomechanical conditions of the lumbar discs. In AIS surgical cohort, Roussouly et al. [6] showed that smaller PI in AIS correlated with better balance and better postoperative result. This probably reflects better biomechanical conditions and higher stability for the remaining mobile segments.

\section{Limitations of the study}

- Spino-pelvic parameters may be more accurately analyzed by the differential analysis of the two components of lumbar lordosis (upper and lower arc) according to the inflexion point, rather than an artificial set of L1S1 lordosis. We did not have such technology for the radiological analysis at the time we started the study. Moreover, we did not measure the thoracic kyphosis in order to simplify the radiographic analysis. Thoracic kyphosis is weakly related to the lumbar lordosis and to none of the pelvic parameters [2]. Therefore, it may not have any influence on the IVD volume variations. The role of thoracic kyphosis will be carefully addressed by another ongoing study focusing on the adjacent segment disease after spinal fusion.
Moreover, thoracic kyphosis measurement on full standing X-ray is of weak reproducibility due to the projection of the shoulders.

- We did not assess the functional status of our patients. In the medical charts, no report of lumbar pain or reduction of daily activity was noticed in the clinical follow-up while these patients were carefully watched annually. None of these patients required additional surgery.

- This study focused on the signal intensity on MRI T2 sequences which specifically explore the nucleus signal intensity and has been shown to be correlated to disc hydration. However, this data is only an indirect sign of disc functional status. Rajasekaran et al. [19] studied the diffusion pattern in the cartilage endplate. Alterations of this zone in MRI were associated with substantial changes in the diffusion pattern of lumbar IVDs in scoliotic patients. The addition of such information to our findings may give a more functional view of the IVD (immediate and long-term effect).

\section{Conclusion}

Although considerable advances have been made in 3D deformity correction after the advent of the Cotrel Dubousset instrumentation, surgeons should pay careful attention to lumbar lordosis and the lumbo-pelvic congruity. In case of inadequate lumbo-pelvic alignment, hydration of the disc may be permanently altered, leading to subsequent degenerative changes. LL should remain congruent with the pelvic morphology especially when extending the fusion to the lower lumbar levels and if PI is high. In the light of these results, it seems that the surgical planning should consider the pelvic morphology to limit the mechanical stress on the discs of the mobile segment underlying the fusion, as patients with high pelvic incidence experienced less disc remodeling after surgery when fusion was extended to the lumbar spine.

\section{Conflict of interest None.}

\section{References}

1. Legaye J, Duval-Beaupère G, Hecquet J, Marty C (1998) Pelvic incidence: a fundamental pelvic parameter for three-dimensional regulation of spinal sagittal curves. Eur Spine J 7:99-103

2. Vialle R, Levassor N, Rillardon L, Templier A, Skalli W, Guigui P (2005) Radiographic analysis of the sagittal alignment and balance of the spine in asymptomatic subjects. J Bone Jt Surg Am 87:260-267

3. Stagnara P, De Mauroy JC, Dran G, Gonon GP, Costanzo G, Dimnet J, Pasquet A (1982) Reciprocal angulation of vertebral 
bodies in a sagittal plane: approach to references for the evaluation of kyphosis and lordosis. Spine 7:335-342

4. Somerville EW (1952) Rotational lordosis; the development of single curve. J Bone Jt Surg Br 34-B:421-427

5. Mac-Thiong JM, Labelle H, Charlebois M, Huot MP, de Guise JA (2003) Sagittal plane analysis of the spine and pelvis in adolescent idiopathic scoliosis according to the coronal curve type. Spine 28:1404-1409

6. Roussouly P, Labelle H, Rouissi J, Bodin A (2013) Pre- and postoperative sagittal balance in idiopathic scoliosis: a comparison over the ages of two cohorts of 132 adolescents and 52 adults. Eur Spine J Suppl 2:S203-S215. doi:10.1007/s00586-012-2571-x

7. Danielsson AJ, Cederlund CG, Ekholm S, Nachemson AL (2001) The prevalence of disc aging and back pain after fusion extending into the lower lumbar spine. A matched MR study twenty-five years after surgery for adolescent idiopathic scoliosis. Acta Radiol 42:187-197

8. Hughes SP, Freemont AJ, Hukins DW, McGregor AH, Roberts S (2012) The pathogenesis of degeneration of the intervertebral disc and emerging therapies in the management of back pain. J Bone Jt Surg Br 94:1298-1304

9. Takashima H, Takebayashi $\mathrm{T}$, Yoshimoto M, Terashima $\mathrm{Y}$, Tsuda H, Ida K, Yamashita T (2012) Correlation between T2 relaxation time and intervertebral disk degeneration. Skeletal Radiol 41:163-167. doi:10.1007/s00256-011-1144-0

10. Green DW, Lawhorne TW 3rd, Widmann RF, Kepler CK, Ahern C, Mintz DN, Rawlins BA, Burke SW, Boachie-Adjei O (2011) Long-term magnetic resonance imaging follow-up demonstrates minimal transitional level lumbar disc degeneration after posterior spine fusion for adolescent idiopathic scoliosis. Spine 36:1948-1954. doi:10.1097/BRS.0b013e3181ff1ea9

11. Violas P, Estivalezes E, Briot J, Sales de Gauzy J, Swider P (2007) Quantification of intervertebral disc volume properties below spine fusion, using magnetic resonance imaging, in adolescent idiopathic scoliosis surgery. Spine 32:E405-E412
12. Setton LA, Chen J (2004) Cell mechanics and mechanobiology in the intervertebral disc. Spine 29:2710-2723

13. Accadbled F, Laffosse JM, Odent T, Gomez-Brouchet A, Sales de Gauzy J, Swider P (2011) Influence of growth modulation on the effective permeability of the vertebral endplate. A porcine experimental scoliosis model. Clin Biomech 26:337-342. doi:10. 1016/j.clinbiomech.2010.11.007

14. Rajasekaran S, Vidyadhara S, Subbiah M, Kamath V, Karunanithi R, Shetty AP, Venkateswaran K, Babu M, Meenakshi J (2010) ISSLS prize winner: a study of effects of in vivo mechanical forces on human lumbar discs with scoliotic disc as a biological model: results from serial postcontrast diffusion studies, histopathology and biochemical analysis of twenty-one human lumbar scoliotic discs. Spine 35:1930-1943. doi:10.1097/ BRS.0b013e3181e9a156

15. Galbusera F, Wilke HJ, Brayda-Bruno M, Costa F, Fornari M (2013) Influence of sagittal balance on spinal lumbar loads: a numerical approach. Clin Biomech 28:370-377. doi:10.1016/j. clinbiomech.2013.02.006

16. Labelle H, Mac-Thiong JM, Roussouly P (2011) Spino-pelvic sagittal balance of spondylolisthesis: a review and classification. Eur Spine J 20(Suppl 5):641-646. doi:10.1007/s00586-011-1932-

17. Hammerberg KW (2005) New concepts on the pathogenesis and classification of spondylolisthesis. Spine 30:S4-S11

18. Roussouly P, Gollogly S, Berthonnaud E, Labelle H, Weidenbaum M (2006) Sagittal alignment of the spine and pelvis in the presence of L5-S1 isthmic lysis and low-grade spondylolisthesis. Spine 31:2484-2490. doi:10.1097/01.brs.0000239155.37261.69

19. Rajasekaran S, Babu JN, Arun R, Armstrong BR, Shetty AP, Murugan S (2004) ISSLS prize winner: a study of diffusion in human lumbar discs: a serial magnetic resonance imaging study documenting the influence of the endplate on diffusion in normal and degenerate discs. Spine 29:2654-2667 